\title{
Welcome/Bienvenue/ Bienvenidos
}

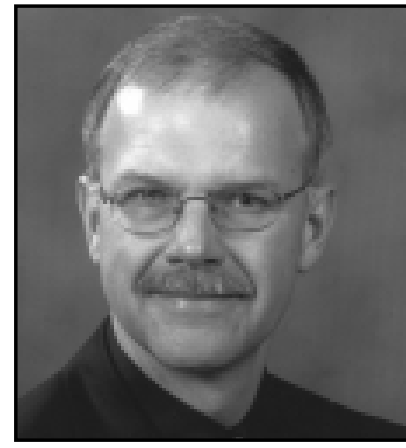

Len Moores, R. P. F.

The Canadian Institute of Forestry / Institute Forestier du Canada (CIF/IFC) is pleased to have this issue of The Forestry Chronicle dedicated to the XII World Forestry Congress - Forests a Source of Life. While the Congress is an opportunity for Canada to showcase its approaches to sustainable forest management, participants will also appreciate the scope of global forest issues and approaches to sustainable forest management. Globally, population growth and loss of forest land are key influences in managing today's forests.

The world population is estimated at six billion people and is expected to reach 9.3 billion by 2050. At today's wood consumption rates, 5.1 billion $\mathrm{m}^{3} /$ year of wood will be required to meet the global population demand by 2050. Current global wood consumption is estimated at 3.3 billion $\mathrm{m}^{3} /$ year, of which $55 \%$ is for fuelwood. Over half of the wood utilized around the world is for fuel. Less than $20 \%$ of the wood harvested in North America and Europe is for fuel wood, while fuelwood harvesting exceeds $60 \%$ of the total wood volume harvested in central America, Africa and Asia.

The increasing world population has many ramifications for forests. Expanding urban centres, conversion of forests for agricultural use, and protecting water quality and quantity are just some of the pressures being placed on a shrinking land base for managing forests. Finding a balance between economic, ecological, social and cultural interests within the context of a decreasing forest land base and society's changing values is the challenge of sustainable forest management.

The CIF/IFC appreciates the significance in understanding these global issues and approaches to sustainable forest management. This special issue of The Forestry Chronicle focusses on Canadian forest management accomplishments, issues and initiatives and future directions. Canada's Prime Minister, The Honourable Jean Chrétien, provides an opening welcome to the delegates attending the Congress. Feature articles were provided by the Canadian Forest Service, Natural Resources Canada-the federal government leads on forest management and research in Canada and from the Canadian Council of Forest Ministers providing an overview of the provincial responsibilities to managing and maintaining Canada's forested areas. The Canadian International Development Agency highlights its priority in supporting developing country forestry programs. Complimenting these articles are papers identifying Canada's role in biodiversity and the environment, industry innovation, private land management, and Aboriginal forestry. Urban forestry is featured as well as articles on Canada's National Forest Strategy and Model Forest Program. As a foundation to Canadian forestry, articles are included on education and on forest practitioners.

We are pleased to present this special issue to our colleagues attending the XII World Forestry Congress, and as President, and on behalf of the CIF/IFC members, I welcome you to this historic event.

Len Moores, R.P.F., President Canadian Institute of Forestry

L'Institut forestier du Canada / The Canadian Institute of Forestry (IFC/CIF) a l'honneur de dédier ce numéro du Forestry Chronicle au XII ${ }^{\mathrm{e}}$ Congrès forestier mondial - La forêt, source de vie. Tandis que le Congrès donne au Canada l'occasion d'exposer les approches privilégiées en faveur d'un aménagement forestier durable, les participants apprécieront également la portée des questions qui se posent à l'échelle du globe et les démarches préconisées dans ce domaine. La croissance démographique et la perte des terres forestières dans le monde sont des influences clés dans la gestion des forêts actuelle.

Selon les estimations, la population mondiale dépasse désormais les six milliards d'habitants et est censée atteindre le chiffre de 9,3 milliards d'ici à 2050. Si la consommation du bois se poursuit au rythme d'aujourd'hui, 5,1 milliards de mètres cubes de bois par an seront alors nécessaires pour satisfaire la demande de la population mondiale. La consommation actuelle de bois dans le monde est estimée à 3,3 milliards de mètres cubes par an, dont 55 pour cent sont destinés au bois de feu. Plus de la moitié du bois utilisé dans le monde est en effet destinée au chauffage. Moins de 20 pour cent du bois récolté en Amérique du Nord et en Europe sont utilisés à cette fin, tandis que cette proportion dépasse 60 pour cent en Amérique centrale, en Afrique et en Asie.

L'augmentation de la population mondiale a de multiples répercussions sur la forêt. L'expansion des centres urbains, la conversion des terres forestières à des usages agricoles et la protection de la qualité de l'eau et de sa disponibilité ne sont que quelques-unes des contraintes qui s'exercent sur des terres qui se rétrécissent au regard de la gestion forestière. Trouver un équilibre entre des intérêts économiques, écologiques, sociaux et culturels dans le contexte d'un territoire forestier qui va en diminuant et les valeurs changeantes de la société, tel est le défi de l'aménagement forestier durable.

L'IFC/CIF en mesure la portée par le regard qu'il porte sur ces enjeux planétaires et sur l'aménagement forestier durable. Ce numéro spécial du Forestry Chronicle souligne les réalisations de la gestion canadienne des forêts et met l'accent sur les questions abordées, les initiatives et les orientations futures. Dans un mot d'ouverture, l'hon. Jean Chrétien, Premier ministre du Canada, souhaite la bienvenue aux délégués présents. Les articles de fond sont communiqués par Forêts Canada, Ressources naturelles Canada - principaux intervenants du gouvernement fédéral dans les domaines de la gestion forestière et de la recherche au Canada, et par le Conseil canadien des ministres des forêts donnant un aperçu des responsabilités provinciales pour la gestion et la préservation des aires boisées du Canada. L'Agence canadienne de développement international met en évidence la priorité accordée au soutien des programmes de foresterie dans les pays en développement. Des articles qui traitent du rôle du Canada dans le domaine 
de la biodiversité et de l'environnement, de l'innovation de l'industrie, de la mise en valeur des terres privées et de la foresterie autochtone viennent compléter ce tableau. La foresterie urbaine est présentée ainsi que des articles consacrés à la Stratégie nationale sur les forêts du Canada et au Programme de forêts modèles. Au fondement de l'engagement forestier du Canada, des articles traitent de l'éducation et des professionnels de la foresterie.

Nous présentons avec plaisir ce numéro spécial à nos collègues qui prennent part au XII ${ }^{\mathrm{e}}$ Congrès forestier mondial et, à titre de président et au nom des membres de l'IFC/CIF, je leur souhaite la bienvenue à cet événement historique.

\section{Len Moores, R.P.F., Président Institut forestier du Canada}

El Instituto Forestal Canadiense / The Canadian Institute of Forestry (CIF) tiene el agrado de dedicar el presente número de su revista The Forestry Chronicle al XII Congreso Forestal Mundial Forests a Source of Life (El bosque: Fuente de vida). El Congreso le brinda a Canadá la oportunidad de presentar los enfoques que ha adoptado en materia de manejo forestal sustentable y al mismo tiempo permite que los participantes aprecien el alcance mundial de los temas forestales y de los enfoques para la gestión sustentable de los bosques. El crecimiento demográfico y la pérdida de áreas boscosas en todo el mundo son las consideraciones esenciales que aborda el manejo actual de los bosques.

Se estima que la población mundial alcanza a seis mil millones de personas y el pronóstico para el año 2050 es de 9300 millones. Si seguimos consumiendo madera al ritmo actual, se calcula que se requerirán 5100 millones de metros cúbicos de madera al año para satisfacer la demanda mundial en el 2050. El consumo actual de madera en el mundo se estima en 3300 millones de metros cúbicos al año, de los cuales 55 por ciento se destina a leña. De hecho, más de la mitad de la madera que se utiliza actualmente en el mundo de destina a combustible. En América del Norte y Europa este uso representa menos del 20 por ciento del volumen total de madera cosechada, mientras que en América Central, África y Asia sobrepasa el 60 por ciento. El crecimiento de la población mundial tiene numerosas repercusiones en los bosques.
La expansión de los centros urbanos, la conversión de áreas arboladas para uso agrícola y la protección de la calidad y cantidad del agua son sólo algunas de las presiones impuestas a una superficie cada vez más reducida para el manejo de los bosques. El desafío del manejo forestal sustentable es encontrar un equilibrio entre los intereses económicos, ecológicos, sociales y culturales en el contexto de una superficie forestal en disminución y los cambiantes valores de la sociedad.

El Instituto Forestal Canadiense está consciente de lo que significa entender estos asuntos de alcance mundial e integrarlos a los enfoques de manejo forestal sustentable. El presente número especial de The Forestry Chronicle destaca los logros de Canadá en el área de manejo forestal, los desafíos actuales y las orientaciones a futuro. El Congreso se inicia con unas palabras de bienvenida del Primer Ministro de Canadá, Honorable Jean Chrétien, a los delegados asistentes. Los artículos de fondo los proporcionó el Servicio Forestal Canadiense (CFS), Ministerio de Recursos Naturales de Canadá (oficina principal del Gobierno Federal en materia de manejo e investigación forestal en Canadá) y el Consejo canadiense de ministros forestales (CCFM), que proporcionó una visión general de las responsabilidades provinciales en el manejo y preservación de las áreas boscosas de Canadá. La Agencia Canadiense para el Desarrollo Internacional subraya la prioridad que asigna al apoyo de programas forestales de los países en desarrollo. Esta visión se complementa con artículos sobre el papel que desempeña Canadá en las áreas de biodiversidad y medio ambiente, innovación de la industria, gestión de tierras privadas y silvicultura aborigen. Otros artículos abordan los temas del espacio forestal urbano, la Estrategia Forestal Nacional de Canadá (NFSC) y el Programa de bosques modelos. Artículos sobre educación y los profesionales del sector forestal destacan el compromiso de Canadá a nivel del sector.

Tenemos el agrado de presentar este número especial de la revista a nuestros colegas que asisten al XII Congreso Forestal Mundial y, como presidente y a nombre de los miembros del Instituto Forestal Canadiense, les doy la bienvenida a este evento histórico.

\section{Len Moores, R.P.F., Presidente Instituto Forestal Canadiense}

The Forestry Chronicle Advisory Board/Comité consultatif

Evelynne Wrangler, R.P.F. (Chair), Director National Initiatives, Canadian Forest Service / co-présidente, directrice, Initiatives nationales, Service canadien des forêts; Allan Masters (Vice Chair), Deputy Minister, Newfoundland Department of Forest Resources and Agrifoods / sous-ministre, Ministère des Ressources forestières, de l'Agriculture et de l'Alimentation, Terre-Neuve; Mike Apsey, R.P.F., Consultant, Forest and Trade Policy / Consultant, Politiques forestières et Commerce; Jean Cinq-Mars, Executive Director, Wildlife Habitat Canada / directeur exécutif, Habitats fauniques Canada; Johanne Gauthier, ing. f., Présidente-directrice générale, Ordre des ingénieurs forestiers du Québec; Dr. Yvan Hardy, ing. f., Assistant Deputy Minister, Canadian Forest Service, Natural Resources Canada / sous-ministre adjoint, Service canadien des forêts, Ressources naturelles Canada; Craig McManus, R.P.F., Consultant; Bruce I. McIntyre, R.P.F., PriceWaterhouseCoopers; Dr. Gilbert G. Paillé, ing. f., President and Chief Executive Officer, FERIC / président directeur général, FERIC; Tony Rotherham, R.P.F., Consultant; Dr. John Saddler, Dean, Faculty of Forestry University of British Columbia / doyen, Faculté de Foresterie, Université de la Colombie-Britannique; Peggy Smith, R.P.F., Professor, Lakehead University / professeur, Université Lakehead.

Associate Editors / Éditeurs Associés L.C. Duchesne, biodiversity / biodiversité; W.H. White, M. Luckert, economics / économie; P. de Groot, entomology / entomologie; P.N. Duinker, environmental issues / enjeux environnementaux; $\mathbf{K}$. Baldwin, forest ecosystem classification; B. Amiro, P.M. Woodard, fire / feux de forêt; Yill Sung Park, forest genetics / génétique forestière; P. Aird, forest history / histoire forestière; T.A. Erdle, forest management / aménagement forestier; P. Marshall, forest management (mensuration) / aménagement forestier (dendrométrie); K. Mallet, forest pathology / pathologie forestière; R.P. Vlosky, forest products / produits forestiers; K.A. Armson, forest soils / pédologie forestière; R.D. Ayling, W.M. Ciesla, international, forestry / foresterie internationale; C. Huor Ung, mensuration (French) and dendrology (French) / dendrométrie (Français) et dendrologie (Français); V. LeMay, modelling and biometry / modélisation et biométrie; R.J. Hall, remote sensing / télédétection; G.F. Weetman, silviculture and ecology / sylviculture et écologie; A. Groot, J-C Ruel, silviculture / sylviculture; Steen Magnussen, monitoring and resource statistics / surveillance et statistiques; G. Hogan, tree physiology / physiologie forestière; L. Couto, tropical forestry / foresterie internationale; F. Bunnell, I.D. Thompson, wildlife management / aménagement faunique; R. Beauregard, wood products / produits de bois 